\title{
artigo
}

Carvalho Silva, T.K.; Moura Silva, T.T.; Menezes, R.S.; Frazão, I.S.; Santos, C.M.; Gonçalves da Silva, A.M.; Gomes, L.M.S.;

Comparação de rastreamento e estado de ansiedade entre estudantes de enfermagem

\section{Comparação de rastreamento e estado de ansiedade entre estudantes de enfermagem}

\author{
Comparison of trace and state of anxiety among nursing students \\ Comparación de rastreo y estado de ansiedad entre estudiantes de enfermería
}

\begin{abstract}
RESUMO
Objetivo: Comparar os níveis de ansiedade entre estudantes do primeiro e último ano do curso de graduação em enfermagem do Centro de Ciências da Saúde da Universidade Federal de Pernambuco. Método: Estudo descritivo, exploratório, com abordagem quantitativa. A coleta de dados foi realizada por meio de entrevista pessoal e plataforma online, além do Inventário de Ansiedade Traço Estadual (IDATE). Resultados: A maioria dos alunos, tanto no início quanto no final da graduação, apresentou ansiedade, traço e estado predominantemente moderados. Conclusão: Nesta amostra não houve diferenças significativas no traço de ansiedade ou estado entre as variáveis estudadas.
\end{abstract}

DESCRITORES: Ansiedade; Estudantes de enfermagem; Qualidade de vida.

\section{ABSTRACT}

Objective: To compare the levels of anxiety among students in the first and last year of undergraduate nursing studies at the Center for Health Sciences of the Federal University of Pernambuco. Method: Descriptive, exploratory study, with a quantitative approach. Data collection was performed through a personal interview and online platform, in addition to the State Trait Anxiety Inventory (IDATE). Results: The majority of students, both at the beginning and at the end of graduation, showed predominantly moderate anxiety, trait and state. Conclusion: In this sample there were no significant differences in the anxiety or state trait between the variables studied.

DESCRIPTORS: Anxiety; Nursing students; Quality of life.

\section{RESUMEN}

Objetivo: Comparar los niveles de ansiedad entre los estudiantes em el primer y último año de estúdios de pregrado em enfermería en el Centro de Ciencias de la Salud de la Universidad Federal de Pernambuco. Método: Estudio descriptivo, exploratorio con abordaje cuantitativo. La recolección de datos se realizó através de una entrevista personal y plataforma en línea, además como el Inventário de ansiedad por rasgos estatales (STAI). Resultados: La mayoría de los estudiantes, tanto al comienzo de la graduación como al final, presentaron ansiedad, rasgos y estado predominantemente moderados. Conclusión: En esta muestra no hubo diferencias significativas en el rasgo de ansiedad o estado entre las variables estudiadas.

DESCRIPTORES: Ansiedad; Estudiantes de enfermería; Calidad de vida.

RECEBIDO EM: 30/09/2020 APROVADO EM: 23/10/2020

\section{Thayná Karollyne Carvalho Silva}

Graduanda de Enfermagem na Universidade Federal de Pernambuco, Brasil - UFPE.

ORCID: 0000-0002-4848-1208

\section{Thassia Thame de Moura Silva}

Enfermeira, Doutoranda do Departamento de Enfermagem na Universidade Federal de Pernambuco, Brasil- UFPE.

ORCID: 0000-0003-1067-7421

\section{Raquel da Silva Menezes}

Graduanda de Enfermagem na Universidade Federal de Pernambuco, Brasil - UFPE.

ORCID:0000-0001-8797-7233 


\section{Iracema da Silva Frazão}

Enfermeira, Professora do Enfermagem na Universidade Federal de Pernambuco, Brasil - UFPE.

ORCID:0000-0002-4690-3753

\section{Cândida Maria dos Santos}

Enfermeira, Professora do Departamento de Enfermagem na Universidade Federal de Pernambuco, Brasil- UFPE. ORCID:0000-0003-4196-7413

Ana Maria Gonçalves da Silva

Enfermeira, Graduada pelo departamento de Enfermagem na Universidade Federal de Pernambuco, Brasil - UFPE. ORCID: 0000-0001-6057-2105

\section{Luana Maria de Santana Gomes}

Enfermeira, Graduada pelo departamento de Enfermagem na Universidade Federal de Pernambuco, Brasil - UFPE. ORCID: 0000-0002-0482-7772

\section{INTRODUÇÃO}

\section{0} $s$ comportamentos ansiosos e os transtornos de ansiedade (TA) vêm aumentando. São vistos por muitos especialistas como o mal do século e podem ser causados por importantes transformações no que diz respeito ao âmbito cultural, social e econômico no qual os indivíduos estão inseridos ${ }^{1}$. Dados da Organização Mundial da Saúde (OMS) indicam que a prevalência do TA no mundo é de 3,6\%. No continente americano esse transtorno mental alcança maiores proporções e atinge $5,6 \%$ da população, com destaque para o Brasil, onde o TA está presente em 9,3\% da população, possuindo o maior número de casos de ansiedade entre todos os países do mundo ${ }^{2}$.

Há dois tipos de ansiedade: traço e estado, a primeira está relacionada a uma disposição individual, relativamente estável como resposta a uma circunstância considerada estressante pelo indivíduo, na qual ele apresenta uma tendência maior em perceber as situações como ameaçadoras. Já a segunda, se define como um estado emocional transitório que pode se modificar ao longo do tempo, em que a pessoa apresenta sentimentos subjetivos de tensão $0^{3,4}$.

A ansiedade pode ser retratada como uma emoção natural, na qual funciona como impulso para o ser humano alcançar seus objetivos. Esse estado emocional pode se tornar excessivo e assim, doentio, repercutindo negativamente na vida do in- divíduo, favorecendo a dificuldade da capacidade de adaptação e enfrentamento ${ }^{5,6}$.

\section{Dados da}

Organização Mundial

da Saúde (OMS)

indicam que a

prevalência do TA no

mundo é de 3,6\%. No

continente americano

esse transtorno mental

alcança maiores

proporções e atinge

5,6\% da população.

Os sinais e sintomas da ansiedade podem se apresentar nas formas psicológicas e somáticas. Alguns sintomas psicológicos da ansiedade em acadêmicos incluem sentimentos de nervosismo antes de uma aula, pânico, esquecimento durante uma avaliação de aprendizagem, sensação de impotência ao fazer trabalhos acadêmicos ou a falta de interesse em uma matéria difícil, por exemplo. Já os sintomas somáticos englobam as palmas das mãos frias, suadas, taquicardia, taquipnéia, dor no estômago e ainda tremores ${ }^{7}$. A ansiedade pode se tornar duradoura com manifestações como fobias, transtorno conversivo, estados dissociativos, obsessões ou compulsões ${ }^{8}$.

Quando a reação ansiosa exacerbada afeta negativamente o comportamento do indivíduo devido à frequência, gravidade ou mesmo persistência desses sintomas, torna-se disfuncional tipificando-se como transtorno de ansiedade (TA $)^{9}$. Transtornos de ansiedade são os mais graves devido maior duração e complexidade dos sintomas ansiosos, sendo disfuncionais e acompanhados de prejuízos ${ }^{10}$. De maneira geral, estudos indicam que os universitários, e em particular os da área da saúde, apresentam altos níveis de estresse e ansiedade. Níveis elevados dessas emoções acarretam prejuízos na qualidade de vida e no desempenho pessoal e profissional do indivíduo ${ }^{11,12}$.

Assim, para melhorar a qualidade da formação acadêmica dos estudantes universitários é importante o conhecimento dos fatores de risco do desenvolvimento desse transtorno. Nessa perspectiva, este estudo teve como objetivo comparar os níveis de ansiedade entre os estudantes do primeiro e último ano de graduação em enfermagem em uma Universidade Pública. 


\section{artigo}

Carvalho Silva, T.K.; Moura Silva, T.T.; Menezes, R.S.; Frazão, I.S.; Santos, C.M.; Gonçalves da Silva, A.M.; Gomes, L.M.S.;

Comparação de rastreamento e estado de ansiedade entre estudantes de enfermagem

\section{MÉTODOS}

O estudo tratou-se de uma pesquisa descritiva, exploratória, com abordagem quantitativa. Foi realizada no Departamento de Enfermagem, vinculado a Universidade Federal de Pernambuco (UFPE), no município de Recife/PE. A população foi constituída por 154 acadêmicos de enfermagem. Os critérios de elegibilidade para o estudo foram: ser estudantes do primeiro e último ano da graduação, que correspondem às turmas do $1^{\circ}, 2^{\circ}, 9^{\circ}$ e $10^{\circ}$ períodos do curso, estar regularmente matriculados e com frequência ativa na academia ou estágios curriculares obrigatórios, para os alunos do último ano.

Foi incluído na pesquisa a totalidade de acadêmicos matriculados nas turmas

Tabela 1 - Características sociodemográficas dos estudantes do $1^{\circ}, 2^{\circ} 9^{\circ} \mathrm{e}$ $10^{\circ}$ períodos. Recife, 2019.

\section{CARACTERÍSTICAS SOCIODEMOGRÁFICAS}

\section{SEXO}

Feminino

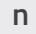

88

Masculino

TOTAL

IDADE

18 a 22

23 a 27

28 a 32

TOTAL

ESTADO CIVIL

Solteiro (a)

95

Casado (a)

TOTAL

LOCAL ONDE RESIDE ATUALMENTE

Casa dos pais

70

Apartamento ou casa alugada com amigos

Casa do estudante

Casa de parentes

Outros

TOTAL

\section{RENDA FAMILIAR}

Menos de 1 salário mínimo

1 salário mínimo

5

12

Até 2 salários mínimos

Mais de 2 salários mínimos

TOTAL

62

31

05

98

98

100

63,26

31,63

5,10

100

96,94

3

3,06

98

100

71,43

9

9,18

3

3,06

8

8,16

8

98

8,16

100

POSSUI FILHOS

Sim

Não

TOTAL

Fonte: Autoras. supracitadas. E excluídos os alunos que não estavam em sala de aula no momento da coleta de dados, que não foram encontrados após três tentativas de contato telefônico e que já tivessem sido matriculados anteriormente em uma mesma disciplina, por entendermos que a experiência já vivenciada por eles poderia alterar os resultados; pessoas desistentes do curso, bem como acadêmicos de menor idade. A coleta foi realizada no período de dezembro de 2018 a abril de 2019 e deu-se presencialmente nas turmas do $1^{\circ}$ ano acadêmico dentro das salas de aula. Já com as turmas do último ano, por se apresentarem em estágio curricular, foi realizada através do Formulário on-line Google. Participaram: 21 alunos do $1^{\circ}$ período; 25 alunos do $2^{\circ}$ período, 26 do $9^{\circ}$ e $10^{\circ}$ períodos. Deste modo, resultou-se em uma amostra total de 98 alunos participantes.

Foi utilizado um questionário sócio-demográfico e os Inventários de ansiedade Traço (IDATE-T) e Estado (IDATE-E). $\mathrm{O}$ primeiro instrumento era composto por questões sócio-demográficas, características da saúde e sobre informações acadêmicas. O IDATE é um instrumento utilizado para quantificar componentes subjetivos relacionados à ansiedade ${ }^{13}$.

Os dados coletados foram digitados no software Epi Info ${ }^{\text {Tw }}$ versão 7.2.3.1 bem como foi formado banco de dados em planilha do Excel', sendo posteriormente analisado pelo software estatístico SPSS ${ }^{\circ}$, versão IBM Statistics 21 através da aplicação de testes estatísticos Qui-quadrado e p-valor exato de Fisher. A coleta de dados foi autorizada pelo comitê através do parecer consubstanciado de número 3.012.179 e CAAE: $99564718.0 .0000 .5208 \mathrm{Na}$ qual os participantes foram esclarecidos sobre os objetivos do estudo e solicitado sua assinatura no Termo de Consentimento livre e esclarecido.

\section{RESULTADOS}

Os dados sociodemográficos dos participantes da pesquisa encontram-se descritos na Tabela 1. 
Foi constatado que $92,85 \%$ dos estudantes não possuem doença crônica. $\mathrm{O}$ questionamento sobre a utilização de medicamentos ou substâncias psicoativas, por ser uma pergunta abrangente não se sabe se os participantes da pesquisa consideraram como substância psicoativa a bebida alcoólica; apesar da maioria (96,98\%) dos acadêmicos desta amostra referir não utilizar, (3,13\%) deles relataram fazer uso de Sertralina e Amitriptilina.

A maioria dos estudantes $(77,55 \%)$ relatou dormir menos de oito horas por noite. Cerca de $(68,04 \%)$ responderam não apresentar dificuldade para dormir, havendo $(31,96 \%)$ deles informado que possuem esse problema, demonstrando como causas prevalentes: insônia, sintomas de ansiedade, preocupação sobre o futuro, excesso de atividades acadêmicas e problemas pessoais.

Analisou-se que a maioria dos estudantes $(75,26 \%)$ acessa a universidade por meio de transporte público. Perguntados sobre a realização de atividades extracurriculares, a maioria (46,39\%) relatou que não participou ou não participa de nenhuma atividade extracurricular, sendo a maioria dos que negaram participar de alguma atividade extracurricular, do primeiro ano acadêmico (54,3\%). Em torno de $82,47 \%$ dos estudantes relataram estar satisfeitos com a escolha do curso, enquanto $17,53 \%$ disseram não estar. Entre os motivos mais prevalentes de tal insatisfação está a não aprovação em cursos de graduação que eram a primeira opção do estudante e a não identificação com o curso de enfermagem.

Sobre as expectativas para a vida pro- fissional, 93,81\% dos estudantes responderam ter boas expectativas e pequena parcela $(6,19 \%)$ relatou ter expectativas ruins. Os motivos para tal eram: falta de desejo em atuar na área e baixo piso salarial da profissão. Houve um predomínio de acadêmicos $(75,26 \%)$ que relataram apresentar sintomas de ansiedade. Ao que se referem aos fatores desencadeadores e/ ou mantenedores dessa ansiedade autodescrita os mais citados foram o excesso de atividades acadêmicas, elaboração de trabalho de conclusão de curso, incertezas do futuro e querer viver o futuro no presente. De acordo com a tabela 1, a maior parte das pessoas do sexo feminino $(77,3 \%)$ e masculino $(70,0 \%)$ apresentou Moderada Ansiedade (MA) do tipo Traço e Estado (71,6\%-mulheres), (60,0\% homens).

Tabela 2 - Análises bivariadas entre ansiedade e características sociodemográficas, acadêmicas e de saúde p-valor do teste Exato de Fisher.

Fator avaliado

\section{Sexo de nascimento}

Feminino

Masculino

TOTAL

p-valor

Faixa etária

18 a 22

23 a 27

28 a 32

TOTAL

\section{Estado civil}

Solteiro

Casado

TOTAL

\section{Padrão de sono}

Dorme mais de 8 horas por noite

Dorme até 8 horas por noite

Dorme menos de 8 horas por noite

TOTAL
Nivel de ansiedade Estado

Baixa

Moderada

Alta

$\begin{array}{cc}23(26,1 \%) & 63(71,6 \%) \\ 4(40,0 \%) & 6(60,0 \%) \\ 98 & \end{array}$

$2(2,3 \%)$

$14(15,9 \%)$

$68(77,3 \%)$

$6(6,8 \%)$

98

$0,565^{1}$

100

$3(30,0 \%)$

$7(70,0 \%)$

$0(0,0 \%)$

98

100

$0,445^{1}$

$17(27,4 \%)$

$45(72,6 \%)$

$0(0,0 \%)$

10(16,1\%)

$47(75,8 \%)$

$5(8,1 \%)$

$8(25,8 \%) \quad 21(67,7 \%)$

2(6,5\%)

$7(22,6 \%)$

23(74,2\%)

$1(3,2 \%)$

$2(40,0 \%)$

$3(60,0 \%)$

$0(0,0 \%)$

$\mathrm{O}(0,0 \%)$

$5(100,0 \%)$

$\mathrm{O}(0,0 \%)$

98

100

98

p-valor

$0,309^{1}$

$26(27,4 \%)$

$68(71,6 \%)$

$1(1,1 \%)$

$17(17,9 \%)$

$72(75,8 \%)$

$6(6,03 \%)$

$1(33,3 \%)$

$1(33,3 \%)$

$1(33,3 \%)$

98

100

$0,049^{1}$

p-valor

$2(66,7 \%)$
$7(36,8 \%)$
$18(23,7 \%)$

$1(33,3 \%)$

$0(0,0 \%)$

$0(0,0 \%)$

$3(100 \%)$

$0(0,0 \%)$

$11(57,9 \%)$

$1(5,3 \%)$

$4(21,1 \%)$

$12(63,2 \%)$

$3(15,8 \%)$

$57(75,0 \%)$

$1(1,3 \%)$

$13(17,1 \%)$

$60(78,9 \%)$

$3(3,9 \%)$

100

98

100 


\begin{tabular}{|c|c|c|c|c|c|c|c|}
\hline \multirow{2}{*}{\multicolumn{8}{|c|}{ Ansiedade autodescrita }} \\
\hline & & & & & & & \\
\hline Não & & $8(33,3 \%)$ & $16(66,7 \%)$ & $0(0,0 \%)$ & $6(25,0 \%)$ & $18(75,0 \%)$ & $0(0,0 \%)$ \\
\hline Sim & & $19(25,7 \%)$ & $57(71,6 \%)$ & $2(2,7 \%)$ & $1(14,9 \%)$ & $57(77,0 \%)$ & $6(8,1 \%)$ \\
\hline \multirow[t]{2}{*}{ TOTAL } & & 98 & & 100 & 98 & & 100 \\
\hline & p-valor & & $0,774^{1}$ & & & $0,238^{1}$ & \\
\hline \multicolumn{8}{|c|}{ Ano Acadêmico } \\
\hline Primeiro ano & & $15(32,6 \%)$ & $31(67,4 \%)$ & $0(0,0 \%)$ & $8(17,4 \%)$ & $33(71,7 \%)$ & $5(10,9 \%)$ \\
\hline Último ano & & $12(23,1 \%)$ & $38(73,1 \%)$ & $2(3,8 \%)$ & $9(17,3 \%)$ & $42(80,8 \%)$ & $1(1,9 \%)$ \\
\hline TOTAL & & 98 & & 100 & 98 & & 100 \\
\hline Fonte: as autoras. & & & & & & & \\
\hline
\end{tabular}

\section{DISCUSSÃO}

$\mathrm{Na}$ amostra em questão, portanto, não houve diferenças significativas ao relacionar a ansiedade com a variável "sexo de nascimento". Contudo, apesar de ter se apresentado com pequena representatividade neste nível de ansiedade, as mulheres apresentaram mais alta ansiedade tanto Traço quanto Estado, em relação aos participantes do sexo masculino.

Ainda, não foi identificada uma disparidade nos níveis de ansiedade com relação à idade dos estudantes bem como em relação ao estado civil, visto que, a maioria deles com idade entre 18 e 22 anos, eram solteiros e apresentaram elevado índice de Moderada Ansiedade (MA) tanto do tipo Traço, quanto do tipo Estado.

Ao realizar a análise bivariada entre ansiedade e estado civil, diante do número pequeno da amostra de indivíduos casados (apenas três de um total de 98 participantes) e nenhum divorciado ou viúvo, não se obteve significância. Como identificado, a maioria dos acadêmicos dormem menos de 8 horas por noite, entretanto nesta amostra, tal fator parece não representar prejuízo nos níveis de ansiedade.

Ao analisarmos o percentual de estudantes que se auto descreveram com ansiedade, em comparação com nível de ansiedade do tipo traço, cujo valor foi de $77,0 \%$, pode-se concluir que diante de fatores ansiogênicos esses indivíduos acreditaram estar com altos níveis de ansiedade, porém, a análise de dados vai de encontro

\section{Ainda, não foi}

identificada uma

disparidade nos

níveis de ansiedade

com relação à idade

dos estudantes bem

como em relação ao

estado civil, visto que,

a maioria deles com

idade entre 18 e 22

anos, eram solteiros $\mathrm{e}$

apresentaram elevado

índice de Moderada

Ansiedade (MA) tanto
do tipo Traço, quanto
do tipo Estado.

à percepção desses alunos, visto que os mesmos estão conseguindo lidar com os fatores desencadeadores de ansiedade de maneira saudável quando percebemos que apresentam moderada ansiedade.

Ao comparar os níveis de ansiedade entre alunos do primeiro e último ano acadêmico foi verificado que houve predominância do nível Moderada ansiedade tanto Traço quanto Estado nas turmas, conforme descrito na tabela ${ }^{4}$. Desta forma, pôde-se perceber que boa parte dos alunos do primeiro ano tem mostrado grande disposição para apresentarem-se ansiosos (com ampla MA Traço 71,7\%), bem como já estavam passando por tal sensação, corroborada pela maior prevalência de MA Estado (67,4\%), tal sensação pode ser descrita como positiva quando relembramos que essa emoção é fisiológica e nos induz a pensar sobre uma possível adaptação que pode estar acontecendo com esses indivíduos.

Considerando a definição de traço de ansiedade, segundo Oliveira et al., $(2016)^{11}$, pode-se dizer que a maioria dos alunos tem tendência moderada de apresentar elevações dos níveis de ansiedade em situações consideradas ameaçadoras. Em níveis adequados, a ansiedade é benéfica, estimulante, propulsora, motivadora e acaba se tornando um elemento importante para a obtenção de resultados satisfatórios no cotidiano do indivíduo ${ }^{14}$.

Em outro estudos realizados com estudantes de enfermagem foi observado que alguns aspectos auxiliam o aluno no enfrentamento da ansiedade, especifica- 
mente no que tange à insegurança e auto-estima. São eles: a boa experiência do docente no acompanhamento de estágios; existência de um serviço de apoio psicológico ao estudante e bom desenvolvimento de aula prática que permita a participação do estudante. ${ }^{15}$

\section{CONCLUSÃO}

De acordo com os resultados, foi possí- vel concluir que nesta amostra não houve nenhuma diferença significativa da ansiedade traço nem estado entre as variáveis estudadas tanto nos estudantes do início bem como do final da graduação. Contudo, o estudo acerca da ansiedade em estudantes universitários é de suma importância, pois contribui para o conhecimento da população em questão, na qual pode favorecer o desenvolvimento de potencialidades, autonomia e adaptação tanto no ingresso ao ensino superior, quanto na saída da universidade.

Em relação às limitações deste estudo, destaca-se a predominância do gênero feminino na amostra, bem como a realização em um único curso da área da saúde e ausência de estudos que abordem alguns resultados encontrados por essa pesquisa. Recomenda-se que estudos futuros apresentem controle de gênero e uma amostra ampla com a inserção de outros cursos da área da saúde.

\section{REFERÊNCIAS}

1.Costa KM, Sousa KR, Formiga PA, Silva WS, Bezerra EB. Ansiedade em universitários na área da saúde. In: II Congresso Brasileiro das Ciências da Saúde; Junho de 2017.

2. Fernandes MA, Ribeiro HK, Santos JD, Monteiro CF, Costa $\mathrm{RD}$, Soares RF. Prevalence of anxiety disorders as a cause of workers' absence. Rev Bras Enferm [Internet]. 2018;71(Suppl 5):2213-20. DOI: http://dx.doi.org/10.1590/0034-7167-20170953

3. Andrade L, Gorenstein C, Vieira Filho AH, Tung TC, Artes R. Psychometric properties of the Portuguese version of the State-Trait Anxiety Inventory applied to college students: factor analysis and relation to the Beck Depression Inventory. Brazilian Journal of Medical and Biological Research. Março de 2001;34(3):367-74. DOI: http://dx.doi.org/10.1590/S0100$879 \times 2001000300011$

4. Gama MM, Moura GS, Araújo RF, Teixeira-Silva F. Trait anxiety in Brazilian university students from Aracaju. Revista de Psiquiatria do Rio Grande do Sul. 2008 Abr;30(1):19-24. DOI: https://doi.org/10.1590/S0101-81082008000100007

5. Alves TC de TF. Depressão e ansiedade entre estudantes da área de saúde. Rev. Med. (São Paulo) [Internet]. 4 de setembro de 2014 [citado 21 de outubro de 2020];93(3):101-5. Disponivel em: http://www.revistas.usp.br/revistadc/article/ view/103400

6. Claudino J, Cordeiro R. Níveis de ansiedade e depressão nos alunos do curso de licenciatura em enfermagem. 0 caso particular dos alunos da Escola Superior de Saúde de Portalegre. Millennium-Journal of Educational, Technologies, and Health. [Internet]. Fevereiro de 2006 [citado 21 de outubro de 2020]; (32):197-210. Disponível em: https://revistas.rcaap.pt/millenium/article/view/8403

7. Carvalho EA, Bertolini SM, Milani RG, Martins MC. < b > Índice de ansiedade em universitários ingressantes e concluintes de uma instituição de ensino superior/Anxiety scores in university entering and graduating students from a higher education institution< b. Cienc. Cuid. Saúde [Internet]. 11 de outubro de 2015 [citado 21 de outubro de 2020];14(3):1290 -1298. Disponível em: http://periodicos.uem.br/ojs/index.php/CiencCuidSaude/article/view/23594
8. Pereira FL, Medeiros SP, Salgado RG, de Castro JN, de Oliveira AM. Anxiety signs experienced by nursing undergraduates/Manifestações de ansiedade vivenciadas por estudantes de enfermagem. Revista de Pesquisa: Cuidado é Fundamental Online. 1 de Julho de 2019;11(4):880-6. DOI: 10.9789/21755361.2019.v11i4.880-886

9. Hamm AO, Richter J, Pané-Farré C, Westphal D, Wittchen HU, Vossbeck-Elsebusch AN, Gerlach AL, Gloster AT, Ströhle A, Lang T, Kircher T. Panic disorder with agoraphobia from a behavioral neuroscience perspective: Applying the research principles formulated by the Research Domain Criteria (RDoC) initiative. Psychophysiology. Março de 2016;53(3):312-22. DOI: https:// doi.org/10.1111/psyp.12553

10. Pérez-Edgar K, Fox NA. Temperament and anxiety disorders. Child and Adolescent Psychiatric Clinics. 1 de Outubro de 2005;14(4):681-706. DOl:https://doi.org/10.1016/j. chc.2005.05.008

11. Oliveira C, Varela A, Rodrigues P, Esteves J, Henriques C, Ribeiro A. Programas de prevenção para a ansiedade e depressão: avaliação da percepção dos estudantes universitários. Interações. 2016;12(42). DOI: https://doi.org/10.25755/ int. 11815

12. Leão AM, Gomes IP, Ferreira MJ, Cavalcanti LP. Prevalência e fatores associados à depressão e ansiedade entre estudantes universitários da área da saúde de um grande centro urbano do Nordeste do Brasil. Revista brasileira de educação médica. Dezembro de 2018;42(4):55-65. DOI: https://doi. org/10.1590/1981-52712015v42n4rb20180092

13. Spielberger CD, Gorsuch RL, Lushene RE. STAI manual for the State-Trait Inventory. Palo Alto. 1970.

14. Lima BV, Trajano FM, Chaves Neto G, Alves RS, Farias JA, Braga JE. Avaliação da ansiedade e autoestima em concluintes do curso de graduação em enfermagem. Rev. enferm. UFPE on line. Novembro de 2017:4326-33. DOI: 10.5205/reuol.2354249901-1-ED.1111201708

15. Santos KD, de Assis MA. Fatores que contribuem para a segurança e insegurança do graduando de enfermagem durante o estágio. Enfermagem Brasil. 15 de Fevereiro de 2017;16(1):410. DOI: http://dx.doi.org/10.33233/eb.v16i1.898 